\title{
Brane new world
}

Some theorists propose that our Universe exists as a slice through multidimensional space. Could this 'brane-world' concept unify gravity with nature's other fundamental forces? Roland Pease reports.

magine a parallel universe in which the three familiar dimensions of space and one of time are replaced by alternative dimensions beyond our experience. Now imagine that multiple universes exist as membranes, or branes, through a multidimensional hyperspace. These additional dimensions could be the size of atoms, or infinitely large. We would never be able to enter them, yet they could have profound effects on the physics of our Universe.

Welcome to brane world, a bizarre territory so far only proved to exist in the thoughts of theoretical physicists. Although it might sound like a fanciful exercise in boggling the minds of lesser mortals, brane theory is in fact a serious attempt to solve the most annoying problem in modern physics: working out how to unify gravity with the other three fundamental forces of nature - electromagnetism, and the strong and weak nuclear forces.

\section{Unequal partners}

The leading explanation of how fundamental particles and forces behave is a theory called the standard model. Its main shortcoming is the difficulty of incorporating gravity on an equal footing with the other known forces. "It's remarkable that gravity, despite being the first to be discovered, is by far the most poorly understood force," says Nima Arkani-Hamed of Harvard University, one of the architects of brane-world physics.

The fundamental problem is that, com- pared with its counterparts, gravity is weak. This can be illustrated by comparing the gravitational attraction between two electrons with their mutual electrostatic repulsion gravity is weaker by a factor of $10^{43}$. ArkaniHamed uses a more familiar image to drive the message home: "An ordinary magnet can lift a pin off a table, even though the entire mass of the Earth is tugging down on this pin, trying to prevent it from being picked up."

Yet current theories of the Universe demand that, for a short period of time immediately after the Big Bang, all four fundamental forces were as one. Under these highly energetic conditions, before the forces split from one another as the Universe cooled, gravity must have had the same strength as its three counterparts.

The leading attempt to reconcile gravity with the other fundamental forces is string theory, in which all fundamental particles are represented as vibrating one-dimensional 'strings'. According to string theory, gravity's strength catches up with other forces if one considers its effects over small enough distances. At a length scale of $10^{-35}$ metres, known as the Planck length, it should be possible to probe the fundamental nature of gravity. And according to the leading version of string theory, if one were to probe space at such scales, an additional seven curled up dimensions would be revealed.

Physicists look for fundamental particles in the debris created when larger particles are

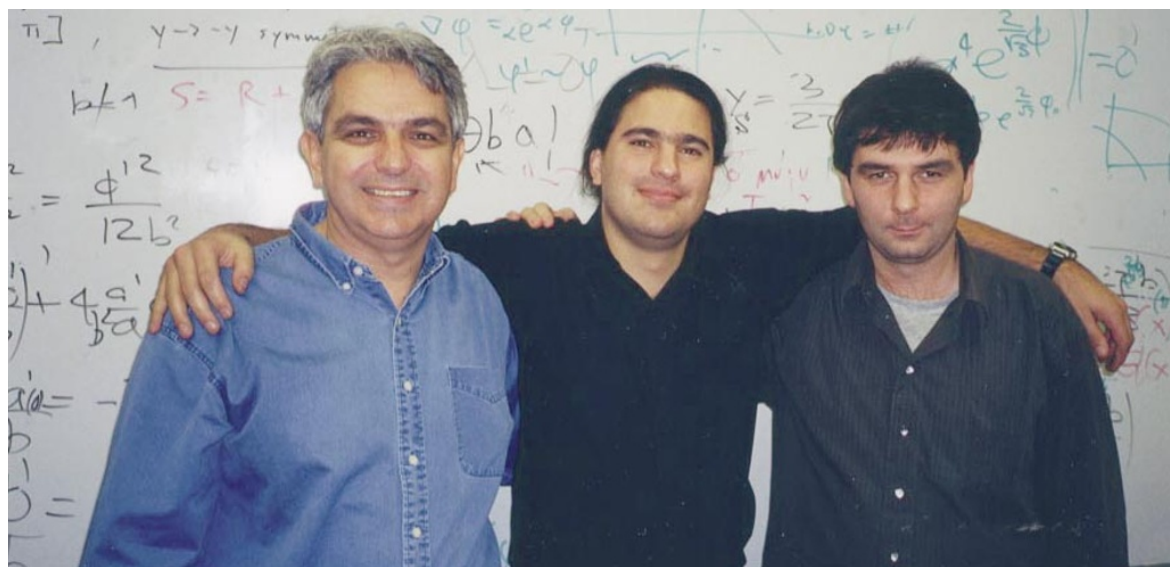

The brains behind the branes: left to right, Savas Dimopoulos, Nima Arkani-Hamed and Gia Dvali. 
Dimopoulos and Gia Dvali turned this approach to the hierarchy problem on its head. Rather than worrying about the inconvenient size of the Planck energy, they wondered what gravity would look like if it too operated at the electroweak scale, making it stronger than we realize.

\section{Distance learning}

Isaac Newton's inverse-square law states that the gravitational pull exerted by an object weakens with distance, $r$, according to the formula $1 / r^{2}$. This is because, as one moves away from a gravitational source, the force's field lines spread out through three dimensions. Arkani-Hamed, Dimopoulos and Dvali started considering scenarios in which our Universe is a brane augmented by additional dimensions, into which gravity could spread. These additional dimensions are not infinite like those of space and time, but of a variety of possible sizes ${ }^{1}$. Dimensions can be finite if they are curled up into closed loops. In essence, the Stanford researchers' proposed dimensions are similar to those of conventional string theory, only larger.

If there is one additional dimension, gravity would obey a $1 / r^{3}$ rule over the length scales covered by this dimension. But to make gravitational effects originate at the TeV scale, this extra dimension has to be so large that its effects on gravity would alter the orbits of the planets - not really a viable option. But with just two extra dimensions, giving a $1 / r^{4}$ law for the length scales of those dimensions, each could be less than a millimetre in size. Anomalous gravitational effects would then only show up at these distances - and that was an intriguing possibility, because physicists were only starting to measure the strength of gravity over sub-millimetre distances (see 'A matter of some gravity', overleaf).

As more dimensions are added, the length scale over which these effects would show up becomes smaller. But even with seven additional dimensions - the same number as in theleading variant of string theory - it would only shrink to about the size of an atomic nucleus. That is a far cry from the $10^{-35} \mathrm{~m}$ of

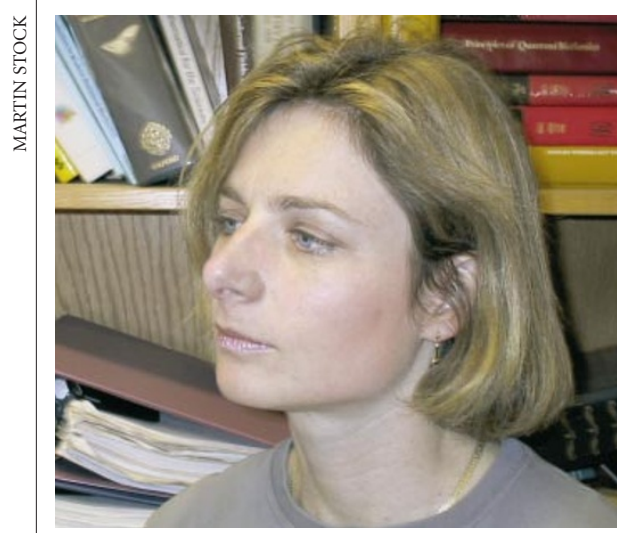

Hidden force: Lisa Randall suggests that gravity may leak into a parallel brane universe.

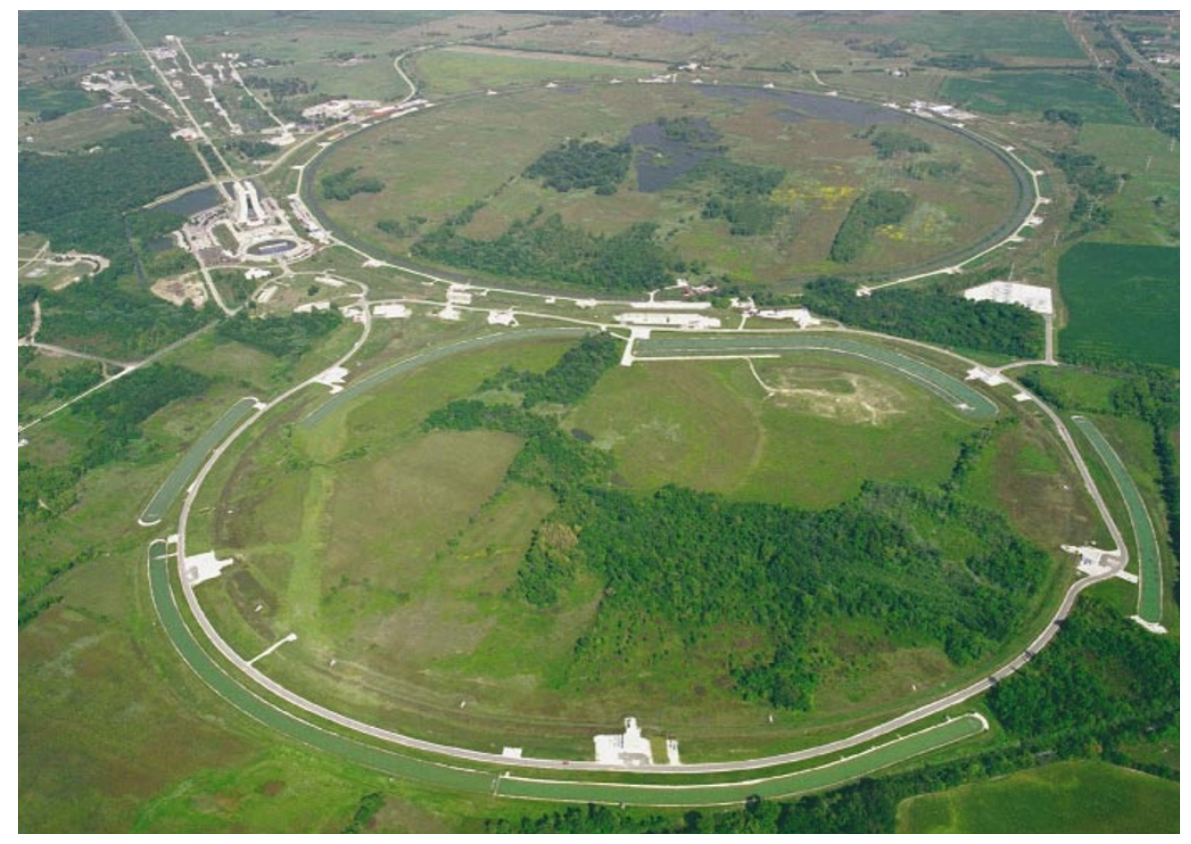

Crash course: the newly refurbished Tevatron will put some variants of brane theory to the test.

the Planck length, and means that it might be possible to probe the nature of gravity using the Tevatron and the next big accelerator, the Large Hadron Collider, scheduled to open in 2006 at CERN, the European Laboratory for Particle Physics near Geneva.

But why should the postulated carrier particles for gravity, known as gravitons, be able to roam through extra dimensions whereas other particles cannot? String theory has an answer. Indeed, the concept of the brane precedes the work of Arkani-Hamed, Dimopoulos and Dvali. According to current formulations of string theory, there are two types of string. The ends of 'closed' strings join up to form a loop in multidimensional space; the ends of 'open' strings do not join up, and must be stuck to a brane.

As Arkani-Hamed, Dimopoulos and Dvali showed with CERN theorist Ignatios Antoniadis ${ }^{2}$, their picture works because gravitons are closed loops and so can wander through the extra dimensions. Other particles are open strings, confined to the three spatial dimensions of our brane Universe.

\section{Warp factor}

The brane-world concept has many guises, depending on how many extra dimensions are included, the size of each of those dimensions, and the different ways of wedding the models to existing theories of high-energy physics. But probably the most significant variant was devised in 1999 by Lisa Randall of Princeton University in New Jersey and the Massachusetts Institute of Technology, and Raman Sundrum, then at Boston University ${ }^{3,4}$.

Randall and Sundrum were toying with brane-world theory in an attempt to iron out niggling problems with supersymmetry. They realized that branes have energy - per- haps a significant amount - and that, according to general relativity, this energy would warp the space around it. They went on to show that this means that gravity could exist in one or more infinitely large extra dimensions without causing significant observed deviations from Newton's $1 / r^{2}$ law. The warping caused by our brane Universe would focus gravity onto the brane and prevent it from getting far away.

In a variation on the theme, Randall and Sundrum have also shown that it is possible to envisage a pair of branes lying almost immediately side-by-side along an extra infinite dimension. One brane, with low energy, is our Universe. The other, hidden, brane is at the Planck energy. This shadow brane then acts like a sump for gravity - it is where gravitons prefer to be and is sufficiently close that they can move from one brane to the other. So, as in the Arkani-Hamed, Dvali and Dimopoulos model, gravity is far stronger than we realize but is dissipated into extra dimensions.

Other variations of brane-world theory are being used to tackle questions about the evolution of the Universe. Astronomers have evidence that there is a mysterious form of energy, known as the cosmological constant, accelerating the expansion of our Universe. Attempts to model this from first principles result in much more rapid expansion than is observed. But brane-world theorists point to a long-forgotten paper $^{5}$ from Valery Rubakov and Misha Shaposhnikov, then at the Institute of Nuclear Research in Moscow. Crudely speaking, this paper argued that the excess energy can be drawn off into extra dimensions, in much the same way as gravity.

One entertaining version of brane-world theory offers an unusual explanation for 'dark matter', the Universe's 'missing' mass that can be detected by its gravitational influence, yet 


\section{ust the possibility
that there could be these extra dimensions out there is so entertaining.}

seems to emit no radiation. Arkani-Hamed, Dvali, Dimopoulos and Nemanja Kaloper, also at Stanford, have proposed that our brane Universe could be folded back on itself (see diagram, right), so that stars at huge distances from us along the brane could be less than a millimetre away along a higher dimension ${ }^{6}$. Their light would not yet have reached us because it has to travel all the way around the folded brane, but their gravity could take the short cut. If this exotic model is correct, then the Laser Interferometer Gravitational-Wave Observatory, with its detectors in Washington state and Louisiana, should detect more intense gravitational waves than are predicted by conventional theories of dark matter.

Once one allows for the existence of more than one brane, it is possible to imagine collisions during the early life of the Universe that would alter the composition of our brane. Dvali, now working at New York University, believes this could explain why our Universe is made solely of matter and has no antimatter, although the two should have been made in equal quantities during the Big Bang. Dvali and Gregory Gabadadze of the University of Minnesota propose that, while the Universe was young, two brane worlds collided and exchanged matter and antimatter". "What we see as an excess of matter on our brane, these other guys see as an excess of antimatter on their brane," says Dvali. In the same paper, Dvali and Gabadadze describe an alternative scenario of 'baby' branes being boiled off our own brane during the Big Bang, carrying away antimatter.

\section{Fertile environment}

The sheer fecundity of brane-world theory is part of its appeal. But its critics complain that the multitude of possibilities it throws up makes it impossible to pin the braneworld theorists down. "The whole approach is not falsifiable," says Gordon Kane, a theoretical physicist at the University of Michigan in Ann Arbor.

Nevertheless, at least some of the predicted brane-world scenarios are now being tested. The range of possibilities, in fact, has already been constrained by observations of Supernova 1987A, which shows that little of its energy could have been released into higher dimensions ${ }^{8}$. That effectively limits the size of the extra dimensions to the sub-millimetre scale. And precise investigations of gravity

\section{A matter of some gravity}

\section{Eric Adelberger and Blayne Heckel of the University} of Washington in Seattle are no strangers to difficult gravity experiments. In the 1980s, they led one of a number of groups that investigated the existence of a postulated fifth force, which would show up as a gravitational anomaly over distances of up to 100 metres. Their findings ${ }^{10}$ helped to kill the idea.

So when they heard about the possible existence of extra dimensions, and that these might reveal themselves as deviations from classical newtonian gravitation over distances of less than a millimetre, it was natural that they would pursue them.

The apparatus they use is a marvel of precision engineering. It consists of a pair of metal discs, one above the other (see right). The upper, aluminium disc is suspended on a fibre of tungsten; the lower, copper disc is spun by a precise motor. A series of holes cut around the perimeter of each disc means that when the lower disc is rotated slowly it exerts little effect on the upper disc with purely newtonian gravity, but any deviations resulting from extra dimensions will cause a just-detectable twist on the upper disc.

The angular movements needed to signal the

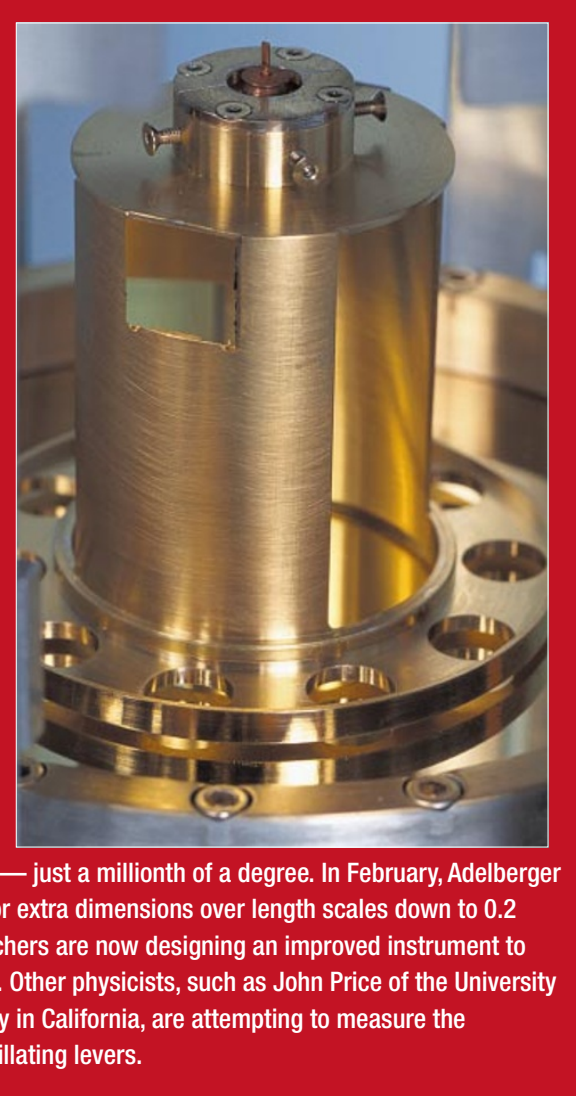

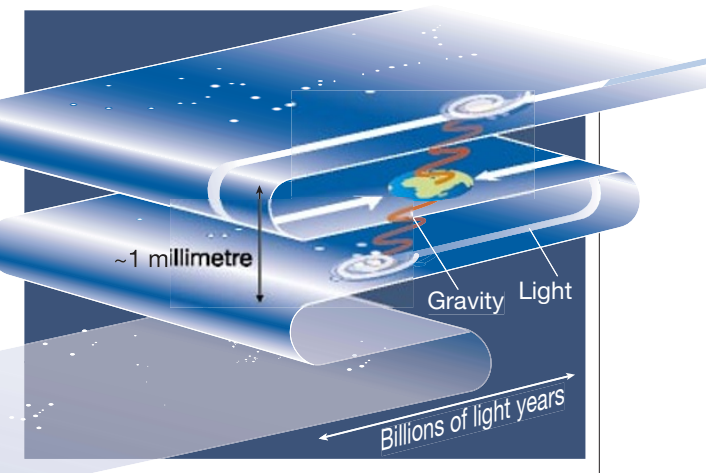

Cosmic origami: a folded brane Universe would allow gravity to slip through an extra dimension.

over these distances are now probing for the extra dimensions predicted by ArkaniHamed and his colleagues (see 'A matter of some gravity', below left).

Meanwhile, high-energy physicists are looking for anomalous results in their experiments that might suggest that gravitons are disappearing into and emerging from the extra dimensions. The most stringent test so far was published in February by the D0 group, working at Fermilab ${ }^{9}$. Researchers led by Greg Landsberg of Brown University in Providence, Rhode Island, re-analysed 60 million particle collisions recorded at the Tevatron between 1992 and 1996, looking for evidence that more pairs of photons, or paired electrons and positrons, were being produced than predicted by the standard model. Additional pairs might be generated by gravitons emerging from an extra dimension, Landsberg explains. His team's negative results suggest that if there are two extra dimensions, they can be no larger than 0.3 millimetres; if there were as many as seven, none can be larger than 2 femtometres $\left(2 \times 10^{-15} \mathrm{~m}\right)$.

The revamped Tevatron, and future colliders, should be able to constrain the braneworld possibilities still further, and may also provide evidence for supersymmetry. But even if these experiments demolish the brane-world's attempts to rival supersymmetry, its enthusiasts seem unlikely to abandon their multidimensional thought experiments. "Just the possibility that there could be these extra dimensions out there is fundamentally so entertaining," says Randall. "It's fun to think about."

Roland Pease is a science producer for BBC Radio.

1. Arkani-Hamed, N., Dimopoulos, S. \& Dvali, G. Phys Lett. B 429, 263-272 (1998).

2. Antoniadis, I., Arkani-Hamed, N., Dimopoulos, S. \& Dvali, G. Phys Lett. B 436, 257-263 (1998).

. Randall, L. \& Sundrum, R. Phys. Rev. Lett. 83, 3370-3373 (1999).

4. Randall, L. \& Sundrum, R. Phys. Rev. Lett. 83, 4690-4693 (1999).

. Rubakov, V. A. \& Shaposhnikov, M. E. Phys Lett. B 125, 139-143 (1983).

6. Arkani-Hamed, N., Dimopoulos, S., Kaloper, N. \& Dvali, G.

J. High Energy Phys. [online] <http:///hhep.sissa.it/archive/papers/ jhep122000010/jhep122000010.pdf0012> (2000).

7. Dvali, G. \& Gabadadze, G. Phys Lett. B 460, 47-57 (1999).

8. Hannestad, S. \& Raffelt, G. G. Phys. Rev. Lett. (in the press).

9. Abbott, B. et al. Phys. Rev. Lett. 86, 1156-1161 (2001).

10. Stubbs, C. W. et al. Phys. Rev. Lett. 58, 1070-1073 (1987). 11. Hoyle, C. D. et al. Phys. Rev. Lett. 86, 1418-1421 (2001). 